\title{
Universal Artificial Intelligence for Intelligent Agents: An Approach to Super Intelligent Agents
}

\author{
Sushil Kumar Singh ${ }^{1}$, Prof. Ashish Kumar Srivastava ${ }^{2}$, Prof. Sitendra Tamrakar ${ }^{3}$ \\ ${ }^{I} M$. Tech. $4^{\text {th }}$ Sem., Computer Science \& Engineering, NRI Institute of Information Science \& Technology, \\ Bhopal, (M. P.), India) \\ ${ }^{2}$ Asst. Prof., CSE Deptt., NRI Institute of Information Science \& Technology, Bhopal, (M. P.), India) \\ ${ }^{3}$ Asst. Prof., CSE Deptt., NRI Institute of Information Science \& Technology, Bhopal, (M. P.), India)
}

\begin{abstract}
Human beings have the real intelligence. The intelligence triggers new thoughts in mind. Human thoughts so many things but he may take long times to solve a complex problem. If he builds such a system which work as like human intelligence, then the time taken to solve the complex problem may be very less. In this case he provides the Artificial Intelligence (AI) to the system. Artificial intelligence based system has the ability to mimic the functions of the human brain. An intelligent agent works on behalf of man. What will happen if send the intelligent agent in new environment? It can work properly or not properly in the new environment. If we provide such intelligence to the agent that it works proper in the new environment without changing their set of rules. Such type of intelligence generally known as Universal Artificial Intelligence (UAI). This paper suggests an idea to build such an intelligent agent that attempts to take the right decision in the new environment. Here we will use the neuro-fuzzy system to provide the more intelligence to agent and this agent can take right decision with learning capability in new environment. If an agent has more intelligence than other agent we can call it super intelligent agent. This paper also shows the simulation of intelligent agent to avoid obstacle in new environment. This simulated intelligent agent shows the good result as compared to existing work.

Keywords- Universal Artificial Intelligence, Hidden Markov Model, Neuro-Fuzzy Systems
\end{abstract}

\section{Introduction}

The main objective is concerns with the behavior of intelligent agents in new environments. How these intelligent agents react in the new environments? Here we try to provide the Universal Artificial Intelligence for intelligent agent i.e. it will work properly in unknown computable environments. Universal Artificial Intelligence based system will work anywhere without changing the rules of that system. Here the Universal Artificial Intelligence based intelligent agent will adept the new environment which are unknown for it and will try to perform the best action for that environment. Here the concept is to make an agent more intelligent i.e. the working of agents just like the best human brain. Such an agent is generally called as a Super Intelligent agent. A super intelligence is associate in nursing intellect that immensely outperforms the simplest human brain in much each field, as well as scientific creative thinking, general knowledge, and social skills. The following basic terms which are the main building blocks for the proposed methodology are as follows:

\subsection{Universal Artificial Intelligence}

Universal artificial intelligence provides the technologies that can drive a car, fly an airplane, play chess, play basketball, or do any human task - no human beings are required to modify the environment of the artificial intelligence rules or change the machine learning algorithm. The foundations of universal intelligence initiate to the origins of philosophy and inductive intellection. Universal engineering correct started with the work of Ray J. Solomonoff at intervals the 1960's. Solomonoff was considering the matter of predicting binary sequences and what he discovered was a formulation for associate inductive mentation system which may be tested to actually apace learn to optimally predict any sequence that features a estimated chance distribution. Not alone is that this theory astonishingly powerful, it put together brings on and elegantly formalizes key philosophical principles behind inductive intellection.

\subsection{Hidden Markov Model (HMM)}

A hidden markov model is AN augmentation of the Markov chain to incorporate observations. A bit like the state transition of the Markov chain, a hidden markov model conjointly includes observations of the state. These observations is also partial in this totally different states can map to an analogous observation and abuzz in that an analogous state can stochastically map to different observations at different times. The assumptions behind a hidden markov model area unit that the state at time $t+1$ solely depends on the state at time $t$, as within the Markov chain. The observation at time $t$ alone depends on the state at time $t$. The 
observations square measure sculptured practice the variable Ot for each time $\mathrm{t}$ whose domain is that the set of potential observations. The concept network illustration of associate within the hidden markov model is represented in one. The concept network is shown for four stages, it'll proceed indefinitely.

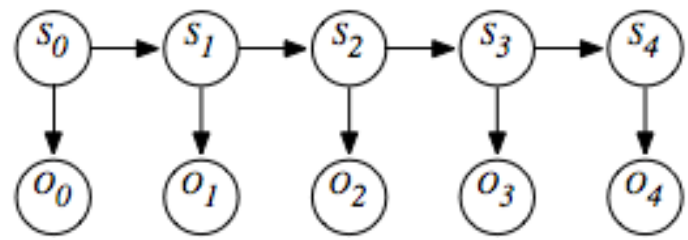

Fig. 1. A Hidden Markov Model as a belief network

A stationary hidden markov model includes the subsequent chance distributions:

- $\mathrm{P}(\mathrm{S} 0)$ specifies initial conditions

- $\mathrm{P}(\mathrm{St}+1 \mid \mathrm{St})$ specifies the dynamics

- $\mathrm{P}(\mathrm{Ot} \mid \mathrm{St})$ specifies the device model

There area unit variety of tasks that area unit common for hidden markov model. The problem of filtering or belief-state observance is to work out the present state supported the present and former observations, particularly to work out,

$\mathrm{P}(\mathrm{Si} \mid \mathrm{O} 0, \ldots, \mathrm{Oi})$.

Note that every one state and observation variables once $\mathrm{Si}$ area unit extraneous as a result of they're not discovered and might be unnoticed once this conditional distribution is computed. The matter of smoothing is to work out state supported past and future observations. Let an agent has observed up to time $\mathrm{k}$ and wants to determine the state at time $\mathrm{i}$ for $\mathrm{i}<\mathrm{k}$; the smoothing problem is to determine

$\mathrm{P}\left(\mathrm{S}_{\mathrm{i}} \mid \mathrm{O}_{0}, \ldots, \mathrm{O}_{\mathrm{k}}\right)$.

All of the variables $S_{i}$ and $V_{i}$ for $i>k$ can be ignored.

\subsection{Neuro-Fuzzy System}

A neuro-fuzzy system could be a fuzzy system that uses a learning algorithmic rule derived from or galvanized by neural network theory to work out its parameters (fuzzy sets and fuzzy rules) by process knowledge samples. Neuro-Fuzzy System combines the benefits of Fuzzy Inference System (FIS) and Artificial Neural Network (ANN), thus it's thought-about as powerful approach in space of Hybrid Intelligent systems to handle complicated real time issues with success. It's like neural net-work with equivalent practicality of fuzzy abstract thought system as a result of it's ability to mix the parallel learning and computation skills of neural network with information illustration and clarification like humans of fuzzy model. This approach will increase the transparency of neural network with infusion of learning capability in fuzzy models.

The neuro fuzzy system is the example of hybrid systems. Hybrid systems are designed as a mix of machine systems and real-world physical components. The machine components are often seen united or additional embedded computers connected by a network and interacting with the physical world via sensors and actuators, with management engineering techniques serving to to coordinate this set-up. The machine a part of the system is so full of the real-world physical part; furthermore, it's to wear down the period properties of the physical world.

\section{Literature Survey}

A research paper in 2001 uses a neuro-fuzzy approach to avoid the obstacle for a mobile robot. Here the approach is able to extract automatically the fuzzy rules and the membership functions in order to guide a mobile robot. The proposed neuro-fuzzy strategy consists of a three-layer neural network along with a competitive learning algorithm. This system has been implemented in simulation obtaining satisfactory results.

A research paper in 2005 uses the hidden markov model for recognition of human motion. A new approach for calculating transition and emission matrices was introduced. Here the observation symbols with fuzzy measurement may be applied in the stochastic model to calculate the real motion primitive sequence. The approach was applied to the human wrist motion to identify the sequence of motion, and the results show that the proposed algorithm has a performance superior to normal hidden markov model when applied to detection of them human wrist motion.

In 2007 a research paper describes the methodology for building the multiagent systems for achieving fault tolerant control system in industry. It uses the concept of fuzzy logic for making the decision. The 
intelligent agents are able to monitor and make diagnosis of possible problems in the process if it uses the concept of fuzzy logic.

\section{Proposed Methodology}

Our proposed methodology is an attempt to provide universal artificial intelligence to agent which can work proper in new environment. Fig. 2 is the schematic diagram of our proposed methodology. It consists the following terms:

- Environment- that the agent occupies, the states that this setting may be in

- Sensors- senses the environment and generate the sensed states/objects

- Hidden Markov Model- generates the sequences or model of sensed states/objects. It also shows the steady states for the dynamic environment

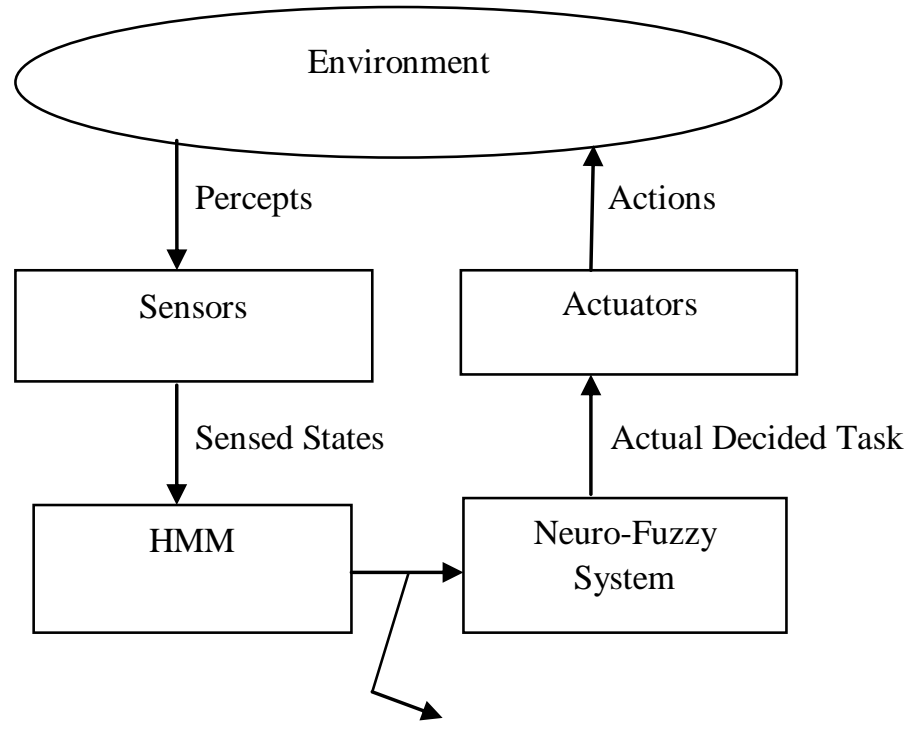

Sequences of Observed States

\section{INTELLIGENT AGENT}

Fig. 2 Proposed model for intelligent agent

- Training and Decision based on Hybrid System (Neuro-Fuzzy System)- generates the actual decided task which are based on training and decision making calculated by hybrid system (neurofuzzy system)

- Actuators- used for performing the action upon the environment

When an agent interact with new environment than it percepts the new environment through sensor it generates the sensed states. The sensed states may be obstacles in front of agent, colors, temperature, other agents, voice etc. The hidden markov model generates the sequences of states with observation. After generating the states the agent make necessary decision and take action upon environment through actuators. The decision and actions are based on hybrid system. Here the neuro-fuzzy system is used in proposed model. A neuro-fuzzy system can be viewed as a three layer neural network. First layer for input variable, the second (hidden) layer for fuzzy rules and third layer for output variable.

\section{Result Analysis}

Here is the simulation of intelligent agent to avoid obstacle in new environment using the proposed methodology. The basic concept is that the agent will sensed the states (obstacle) in new environment and making appropriate angle it will avoid the obstacle.

\subsection{Observations of states using Hidden Markov Model}

After sensing the obstacle through sensors, the hidden markov model generates the sequences of states (obstacles). The fig. 3. Shows the result of observations for hidden markov model. 

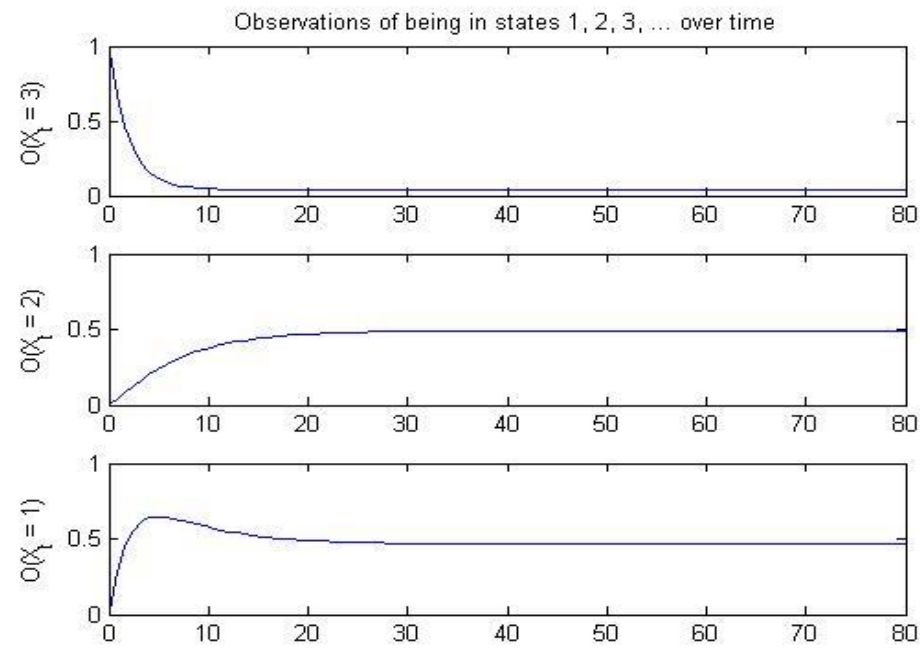

Fig. 3. Observation Result of hidden markov model

\subsection{Add the Fuzzy Rules to Making Appropriate Angle for Avoiding Obstacle}

The agent encompasses a detector which will notice the gap from objects. Detector knowledge provides the indication of a distance from associate degree object, we are able to apply the mathematical logic on those knowledge to get the escaping angle at rational position. Fuzzy may be a smart approach to issues that involve uncertainty, angle management to flee obstacle, rate management, and vision system. Groups of people create choices supported rules. Even if, we tend to might not remember of it, all the selections we tend to create area unit supported laptop like if-then statements. Mathematical logic incorporates an easy, rule-based IF X AND Y THEN Z approach to a resolution management drawback instead of trying to model a system mathematically. Fig. 4 shows the FIS in agent and Fig. 5 shows the input parameters in agent.

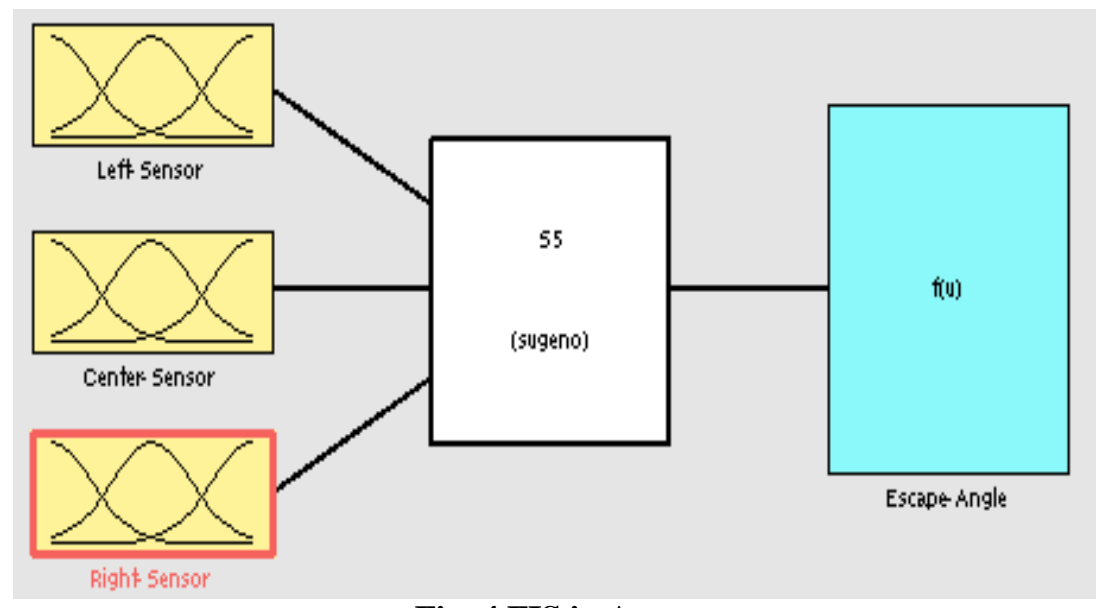

Fig. 4 FIS in Agent

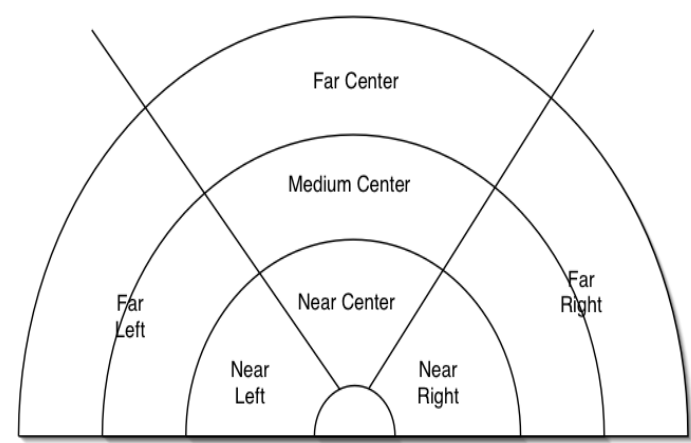

Fig. 5 Input parameters in agent: Left, Right and Center 
The fuzzy variables on input parameters in agent are: close to and much on Left and Right parameters, and near, middle and much for Center parameter. The agent has seven fuzzy rules as below:

1. IF right is far and center is near and left is near THEN angle is RRR.

2. IF right is near and center is near and left is far THEN angel is LLL.

3. IF right is far and center is medium and left is near THEN angle is RR.

4. IF right is near and center is medium and left is far THEN angle is LL.

5. IF right is far and center is far and left is near THEN angle is R.

6. IF right is near and center is far and left is far THEN angel is $\mathrm{L}$.

7. IF right is far and center is far and left is far THEN angle is straight.

Fig. 6 shows the result in MATLAB and fig.

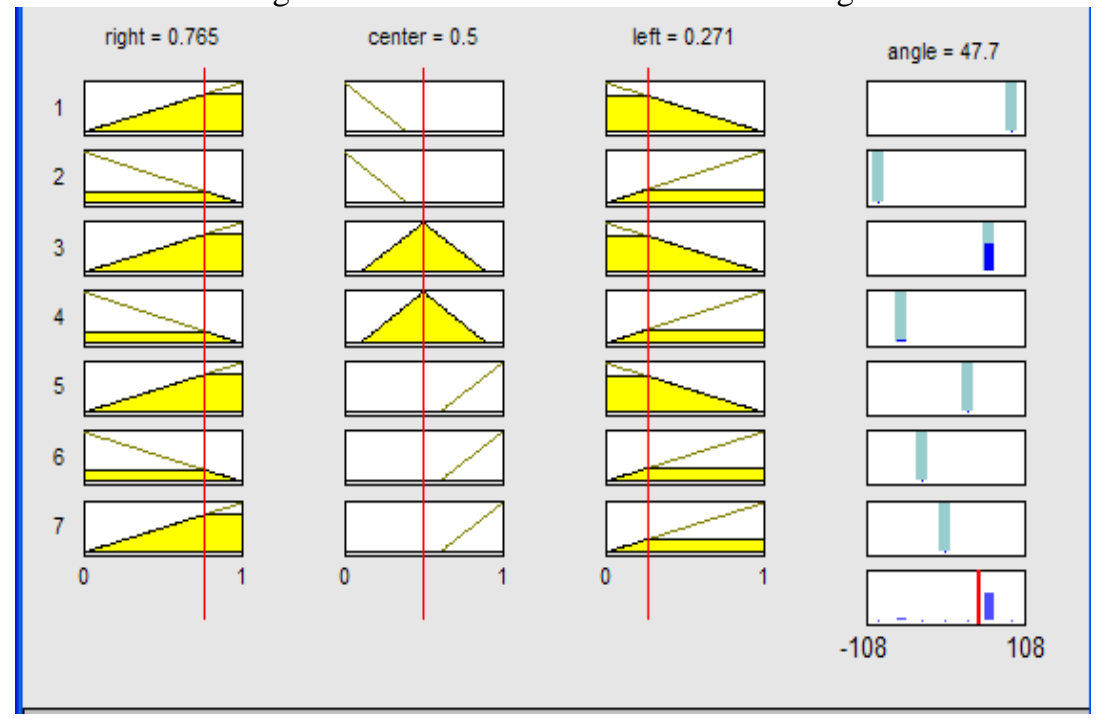

Fig. 6 Result in MATLAB

\subsection{Comparison with existing work and Proposed Methodology}

The table 1 shows the comparison with existing work and the proposed methodology which are differ in some factors.

Table 1. Comparison Table for Existing Work and Proposed Methodology

\begin{tabular}{|c|l|l|l|}
\hline $\begin{array}{c}\text { S. } \\
\text { No. }\end{array}$ & \multicolumn{1}{|c|}{ Factors } & \multicolumn{1}{|c|}{ Existing Work } & \multicolumn{1}{|c|}{ Proposed Methodology } \\
\hline 1. & Working Capability & Based on fixed commands & Based on rules \\
\hline 2. & $\begin{array}{l}\text { Decision Making } \\
\text { Power }\end{array}$ & $\begin{array}{l}\text { Has no decision making } \\
\text { power }\end{array}$ & $\begin{array}{l}\text { Has good decision making } \\
\text { power }\end{array}$ \\
\hline 3. & Learning Capability & Has no learning capability & Has good learning capability \\
\hline 4. & Updating Capability & Has no updating capability & Has updating capability \\
\hline 5. & Efficiency & Average & Very good \\
\hline
\end{tabular}

\subsection{Discussion}

We think that increase the quantity of input variables and membership functions will build the result additional correct. However, we should always not forget that fuzzy inference system should be easy and simple to grasp. Will increase the quantity of variables additionally will increase the quality of fuzzy inference system.

\section{Conclusion}

Mostly intelligent agents work properly which are designed for the known environment. The inbuilt functions are designed for these agents, on the basis they work. Although the intelligent agents work on behalf of man. Suppose we design an intelligent agent for the earth and it contain the features which will proper work for earth and sudden we send it at the other space then how it will react? Imagine! Will it work properly or not? So here this paper tries to build such an intelligent agent which will work for new environment. If it will success then it may solve many complex problems. We can apply the same concepts for the autonomous taxi driver and this autonomous taxi will work for different countries where each country has their own rules for driving i. e. the 
new environments for the taxi. Then this taxi will self learn for unknown environment and then it will take the appropriate action for performing the task. Here we simulate the intelligent agent to avoid obstacle in new environment. Based on the same concept we can simulate other intelligent agent for other task for a new environment and it will show the good result as compared to existing work.

\section{Acknowledgments}

Sushil Kumar Singh would like to thanks his guide Prof. Ashish Kumar Srivastava and co-guide Prof. Sitendra Tamrakar in writing this paper and giving the valuable suggestion.

\section{References}

[1] Juan José Serrano, Arnulfo Alanis Garza, , Rafael Ors Carot, José Mario García-Valdez, Hector Arias, Karim Ramirez and Jose Soria, -Monitoring and Diagnostics with Intelligent Agents using Fuzzy Logic, Advance on-line publication: fifteen August 2007.

[2] Serban Gabriela, "A Reinforcement Learning Intelligent Agent”, Studia Univ. Babes-Bolyai, Informatica, 2001.

[3] Raymond J. Hickey, Christopher J. Hanna, Michaela M. Black and Darryl K. Charles, "Modular Reinforcement Learning Architectures for Artificially Intelligent Agents in Complex Game Environments", IEEE Conference on Computational Intelligence and Games, 2010

[4] Eleni Mangina, "Intelligent Agent-Based Monitoring Platform for Applications in Engineering", International Journal of Computer Science and Applications, 2005.

[5] Waleed H. Abdulla, Kevin I-Kai Wang, and Zoran Salcic, "Multi-agent Software Control System with Hybrid Intelligence for Ubiquitous Intelligent Environments", Springer-Verlag Berlin Heidelberg, 2007.

[6] F. Naghdy \& X. Zhang, "Human Motion Recognition through Fuzzy Hidden Markov Model”, University of Wollongong, Research Online.

[7] Kowalski Robert, "The Logical Way to Be Artificially Intelligent”, Imperial College London.

[8] L. Moreno, G.N. Marichal, L. Acosta, , J.A. M-endez, J.J. Rodrigo, M. Sigut, “Obstacle avoidance for a mobile robot: A neurofuzzy approach", Fuzzy Sets and Systems, 2001, Elsevier.

[9] Zhen Liu , German Florez-Larrahondo, Yoginder S. Dandass, Rayford Vaughn and Susan M. Bridges, “Integrating Intelligent Anomaly Detection Agents into Distributed Monitoring Systems", Journal of Information Assurance and Security 1 (2006).

[10] Browning B., Argall B., \& Veloso M. 2007, "Learning by demonstration with critique of a human teacher", In Proceedings of the Second ACM/IEEE International Conference on Human Robot Interaction.

[11] Jenkins O. \& Grollman D. 2007, "Dogged learning for robots", In IEEE International Conference on Robotics and Automation.

[12] Veloso M. \& Chernova S. 2008,"Multi-thresholded approach to demonstration selection for interactive robot learning”, In Proceedings of the Third ACM/IEEE International Conference on Human-Robot Interaction.

[13] Elaine Rich \& Kevin Knight, “Artificial Intelligence”, McGraw-Hill.

[14] D. McDermott \& E. Charniak, "Introduction to Artificial Intelligence", Pearson Education.

[15] G.A. Vijayalakshmi Pai \& S. Rajsekaran, "Neural Networks, Fuzzy Logic and Genetic Algorithm: Synthesis and Applications", Prentice Hall of India.

[16] Siman Haykin,"Neural Netowrks",Prentice Hall of India.

[17] Timothy J. Ross, "Fuzzy Logic with Engineering Applications", Wiley India. 